\title{
Two heterologously expressed Planobispora rosea proteins cooperatively induce Streptomyces lividans thiostrepton uptake and storage from the extracellular medium
}

\author{
Anna Giardina1', Rosa Alduina1', Elvira Gottardi², Valentina Di Caro ${ }^{13}$, Roderich D Süssmuth² and Anna M Puglia*1
}

\begin{abstract}
Background: A bacterial artificial chromosomal library of Planobispora rosea, a genetically intractable actinomycete strain, was constructed using Escherichia coli-Streptomyces artificial chromosome (ESAC) and screened for the presence of genes known to be involved in the biosynthesis of antibiotics.

Results: One clone with a $40 \mathrm{~kb}$ insert showed antimicrobial activity against Gram positive bacteria. Insert sequence analysis and subcloning experiments revealed that the bioactivity was due to a $3.5 \mathrm{~kb}$ DNA fragment containing two open reading frames. These orfs encode two proteins with high similarity to a putative membrane protein of Streptomyces coelicolor and to the nogalamycin resistance protein SnorO of Streptomyces nogalater, respectively. The role of these two Orfs is unknown in Planobispora. Disruption and complementation experiments revealed that both proteins are necessary for the antibacterial activity and chemical analysis demonstrated that the antibiotic activity was due to thiostrepton, antibiotic used as recombinant clone selection marker.
\end{abstract}

Conclusion: Two Planobispora rosea orfs are responsible for increasing intracellular amounts and storage of thiostrepton in Streptomyces lividans.

\section{Background}

Thiostrepton is a potent thiopeptide antibiotic, widely used as a selection marker for thiostrepton-resistant $(t s r)$ vectors in Streptomyces lividans but its routine use revealed several unexpected biological activities. Remarkably, it can induce resistance to antibiotics having different cellular targets including daunorubicin, sparsomycin, tetranactin and GE2270A [1] and causes expression of thiostrepton-induced proteins [2]. Two of these thiostrepton-induced proteins, TipAL and TipAS, were demonstrated to be alternative in-frame translation products of the tipA gene [3]. In particular, they share the Cterminal region containing the binding domain for thiostrepton and similar cyclic thiopeptide antibiotics [1]. Notwithstanding TipA was initially assigned as a thiostrepton-induced activator of its own transcription

* Correspondence: ampuglia@unipa.it

1 Dipartimento di Biologia Cellulare e dello Sviluppo, Università di Palermo, Viale delle Scienze, Ed.16, 90128 Palermo, Italy

Full list of author information is available at the end of the article
[1], its role has not been deeply understood yet. In fact, tipA has been found to be present in many non-thiopeptide producing strains [4] and it is unclear what metabolic signals are the genuine inducers of its expression. Recently, it was established that thiostrepton is derived from the precursor TsrA, a genetically encoded peptide, suggesting that thiopeptide antibiotics are ribosomally synthesized [5-8].

Heterologous expression systems were successfully applied to synthesize metabolites, produced by hard-tomanipulate strains [9]. Previous results [10-12] have shown that E. coli-Streptomyces artificial chromosomes (ESACs), carrying large inserts of actinomycete DNA, can be introduced into a genetically accessible strain such as Streptomyces lividans, where they are stably maintained as an integrated form in its chromosome.

Planobispora rosea is a genetically intractable actinomycete producer of the thiazolylpeptide antibiotic 
GE2270, a potent inhibitor of the bacterial elongation factor $\mathrm{Tu}$, which is structurally similar to thiostrepton [13].

In this paper we show that two $P$. rosea orfs, encoding a membrane protein and an $\mathrm{ABC}$ transporter, when cloned in Streptomyces lividans, determine thiostrepton uptake and storage from thiostrepton-containing medium. This has been experimentally shown by HPLC-ESI-mass spectrometry, HPLC-UV-DAD, feeding experiments with ${ }^{13} \mathrm{C}$-labeled cysteine and bioassays.

\section{Results \\ Cloning and sequence analysis of a Planobispora rosea 40 kb DNA fragment}

Four clones of Streptomyces lividans, SL-40, -48, -85, 120 , isolated from a $P$. rosea genomic library [11], showed antibacterial activity against $M$. luteus. Since SL-40, which carried the smallest insert ( $40 \mathrm{~Kb})$, was still able to show antibacterial activity, this construct was used for the subsequent investigations. Therefore, the insert was sequenced (GenBank sequence accession number: EU908202). The sequence analysis revealed thirty putative ORFs (Fig. 1a): ten hypothetical proteins, four putative regulators, six proteins related to membrane transport (three $\mathrm{ABC}$-transporters and three membrane proteins), a chitinase, an isomerase, a xanthine dehydrogenase, a terpene synthase, a Ser-Thr-kinase, a Tyr-Serphosphatase, a gamma-glutamyltransferase, an acyl-CoA dehydrogenase, a hydrolase and an oxidoreductase. Since sequence analysis did not reveal the presence of any gene assignable to an antibiotic biosynthesis pathway, different fragments of the $40 \mathrm{~kb}$ insert were subcloned into the ESAC vector and the obtained nine subclones were repeatedly analysed for antibacterial activity against $M$. luteus. Bioassay revealed that only one Streptomyces lividans subclone, named SL-3.5 and carrying a $3.5 \mathrm{~kb}$ fragment, showed antimicrobial activity. Furthermore, it was found that SL-3.5 mycelium inhibited also the growth of Bacillus subtilis, but not of Gram-negative bacteria, as $E$. coli or Pseudomonas aeruginosa (data not shown). Swissprot analysis of the insert sequence indicated that this DNA fragment contained two divergently transcribed orfs, named $a b c$ and imp. imp consists of $1023 \mathrm{bp}$ and the deduced product is a polypeptide consisting of 340 amino acids with $50 \%$ similarity to a membrane protein of S. coelicolor (SCO5138). abc consists of $2430 \mathrm{bp}$ and encodes a protein of 809 amino acids that shows high levels of similarity (80\%) with an ABC-transporter of Streptomyces nogalater involved in nogalamycin resistance (SnorO).

\section{Both imp and abc are involved in antibacterial activity of SL-3.5}

To investigate the role of Imp and Abc in stimulating antibacterial activity in Streptomyces lividans, disruptants containing solely imp or $a b c$ were generated by inser- tional inactivation and named SL-Imp and SL-Abc (Fig. 1b). Bioassays showed that the single knockout mutants were not active against $M$. luteus, indicating that the expression of both imp and $a b c$ is mandatory for antibacterial activity (Fig. 1c).

To confirm that the disruption of imp and $a b c$ genes was the sole reason for the loss of antibacterial activity, complementation mutants were generated and investigated using the antibacterial assay. Our results indicated that, comparably to SL-3.5, both complementation strains had a high inhibitory activity on M. luteus (Fig. 1c).

\section{The antibacterial activity of SL-3.5 is determined by thiostrepton}

Streptomyces lividans ZX7 carries the actinorhodin, undecylprodigiosin and calcium-dependent antibiotic biosynthesis gene clusters in its genome, but they are produced only under particular nutritional conditions [14]. In the conditions we used (growth in $\mathrm{R}_{2}$ YED for 4 days at $30^{\circ} \mathrm{C}$ ) we did not detect any antibacterial activity of S. lividans. To investigate if Planobispora proteins were able to activate either act or red gene expression, spectrophotometric assays of SL-40, SL-3.5 and control strain (SLESAC) were carried out. Our results showed that the amount of Act and Red produced from SL-40 and SL-3.5 was equal to those produced by $S$. lividans and SL-ESAC (data not shown). Furthermore, SL-40 and SL-3.5 failed to produce the calcium dependent antibiotic CDA.

To identify the antibacterial substance, dried methanolic extracts of SL-3.5 were extracted with a mixture of water:acetone:ethyl acetate $(1: 1: 1)$ and both phases analyzed by HPLC-ESI-MS analysis. The upper phase of SL3.5 extract contained a large peak $\left(t_{R}=7.0 \mathrm{~min}\right)$ which was absent in the SL-ESAC sample (data not shown). To our surprise, the UV-spectrum as well as the molecular mass $\left([\mathrm{M}+\mathrm{H}]^{+}=1664.5 \mathrm{Da}\right)$ identified the active substance as thiostrepton (data not shown). Comparison with commercially available thiostrepton confirmed this result. Moreover, TLC analysis revealed that also the activity of SL-40 was exclusively due to thiostrepton (data not shown).

SL-3.5 accumulates thiostrepton during antibiotic selection As thiostrepton was used as selection marker for ESAC containing S. lividans strains and, at the time of this work, the thiostrepton gene cluster had not been identified yet, two hypotheses arose from our results. The first was the presence of silent thiostrepton biosynthetic genes in S. lividans, whose expression was activated by Planobispora proteins and the second one was the uptake and storage of thiostrepton from the medium during SL-3.5 growth under selection pressure and its subsequent release into thiostrepton-free medium. 

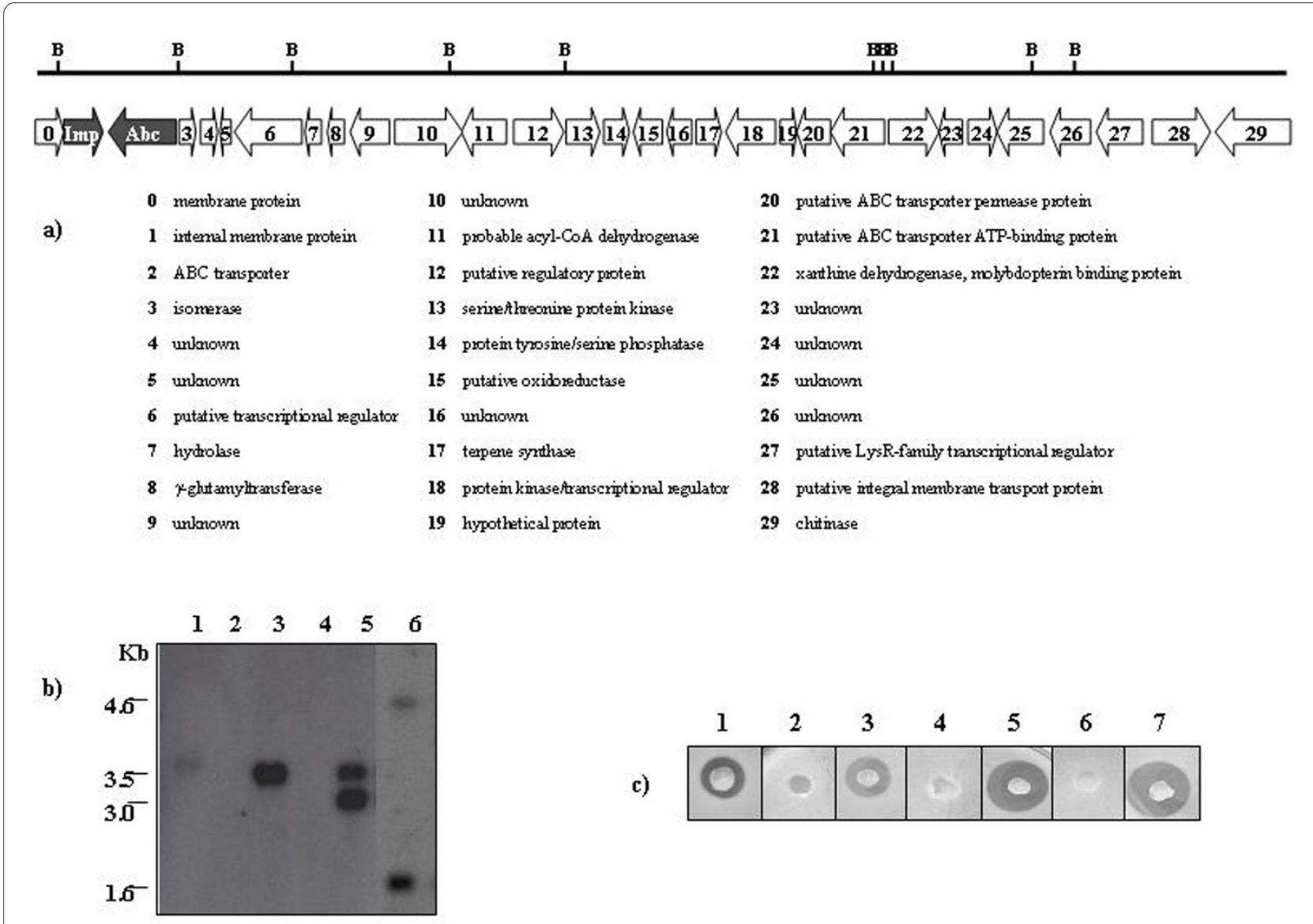

c)

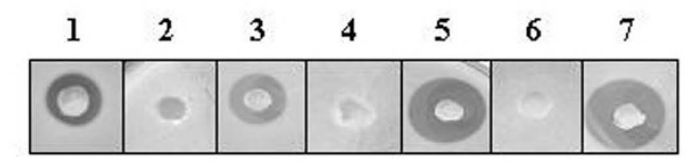

Figure 1 Sequence analysis of the SL-40 insert and Bioassay. a) Schematic diagram of sequence analysis of clone SL-40. Putative ORFs are indicated by the arrows; below is a list of the protein which each ORF is most similar to. The genes involved in SL-3.5 antibacterial activity are shown in grey and the remaining ORFs are in white. B indicates BamHI sites. b) Southern blot analysis of BamHI-digested total DNA isolated from SL-3.5 (lane 1), S. lividans (lane 2), P. rosea (lane 3), SL-ESAC (lane 4), SL-Abc (lane 5) and SL-Imp (lane 6). The BamHI fragment of 3.5 Kb (derived from digestion of ESAC3.5) was used as probe. c) Bioassay against Micrococcus luteus: 1) SL-40; 2) SL-ESAC; 3) SL-3.5; 4) SL-Abc; 5) SL-Abc/Imp; 6) SL-Imp and 7) SL-Imp/Abc.

To investigate whether a de novo thiostrepton synthesis had occurred in S. lividans, we carried out feeding experiments using isotopically labelled $(R, S)-\left[3-{ }^{14} \mathrm{C}\right]$ cysteine as substrate. The radioactive samples were analyzed by TLC followed by bioassay and autoradiographic analysis. The bioassay clearly demonstrated that the inhibition halo generated by SL-3.5 on M. luteus corresponded to the TLC spot with $\mathrm{R}_{\mathrm{f}}=0.8$, identical to that one of thiostrepton produced from S. laurentii (Fig. 2a). However, the autoradiographic analysis revealed that only the antibiotic produced by $S$. laurentii is radioactive (Fig. 2b). Therefore, cysteine was not used by S. lividans as a substrate for the biosynthesis of the previously identified thiostrepton.

In a subsequent set of experiments we investigated if the two $P$. rosea proteins, when present in SL-3.5, caused the uptake of the thiostrepton used for strain selection. Hence, we monitored the presence of the antibiotic in the mycelial DMSO extracts of SL-3.5 after one (I), five (V) and ten (X) passages of growth in thiostrepton-free $\mathrm{R}_{2}$ YED medium by antibacterial bioassay (Fig. 3a). In addition, the same amount of each extract was spotted on a cover glass and photographed under UV light, as thiostrepton results luminescent (Fig.3b).

Thiostrepton was detected being released from SL-3.5 after passage I until passage V but could not be detected after passage X. As a positive control S. laurentii extracts were used and thiostrepton could always be detected upon extraction. As a negative control SL-ESAC was used, which was unable to take up and store thiostrepton. All these results suggest that the thiostrepton is not synthesized de novo from SL-3.5 but it is accumulated in the cells from the thiostrepton-containing medium. The detected antibacterial activity is due to the release of thiostrepton during subsequent growth of SL-3.5 on thiostrepton-free medium. 

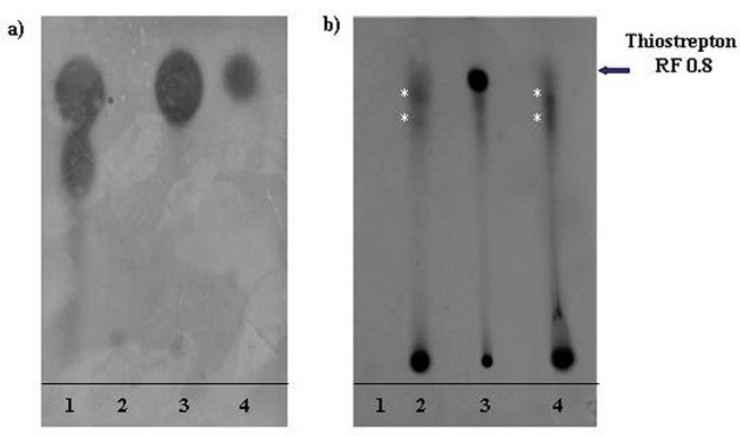

Figure 2 Feeding experiments. (a) Thin Layer Chromatography followed by bioassays on M. luteus and (b) autoradiography showing radio-labeled thiostrepton (1); SL-ESAC (2), S. laurentii (3) and SL-3.5 (4) radioactive extracts. Solvent: acetone. Asterisks indicate the substance present in SL-ESAC and SL-3.5 that are not active and are different from thiostrepton.

\section{Thiostrepton enters the cells in SL-3.5}

To investigate whether thiostrepton was membranebound or cytoplasmic, we analysed the expression level of TipAS and the occurrence of TipAS-thio (thiostreptonbound TipAS) as Murakami et al. [3] demonstrated thiostrepton activates tipA expression and binds TipAS. Therefore, proteins extracted from SL-3.5 grown in presence or absence of thiostrepton for two passages in the culture medium were analyzed by 2D-DIGE and compared to the negative control SL-ESAC unable to store thiostrepton (Fig. 4). As expected [15], our results showed that expression of TipAS and TipAS-thio in the negative control SL-ESAC is 10 - and 7-fold higher in presence of thiostrepton rather than in its absence. Interestingly, the protein analysis revealed that TipAS and TipAS-thio are 20- and 10-fold more expressed in SL-3.5 than in the negative control SL-ESAC grown in thiostrepton absence. In addition, externally added thiostrepton only slightly induced TipAS and TipAS-thio expression in SL-3.5, suggesting that thiostrepton was already present

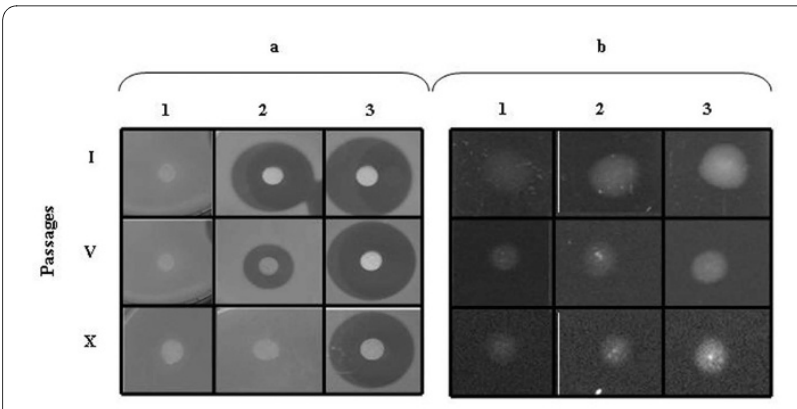

Figure 3 Bioassay on M. luteus (a) and UV visualization (b): extracts were collected from SL-ESAC (1), SL-3.5 (2) and S. laurentii (3), grown for I, $\mathrm{V}$ or $\mathrm{X}$ passages in thiostrepton-free medium. in the cells of SL-3.5 after the passage II in thiostreptonfree $\mathrm{R}_{2} \mathrm{YED}$ medium.

\section{Imp and $\mathrm{Abc}$ are involved in thiostrepton uptake}

In order to test if Imp and Abc are involved in thiostrepton uptake or secretion, we performed quantitative analysis by HPLC/UV-DAD/ESI-MS of mycelium extracts of SL-3.5, SL-Abc and SL-Imp after two passages in a thiostrepton-free medium. HPLC ESI-MS analysis revealed (Fig. 5) that both Orfs are essential for the uptake of the antibiotic, in fact while negative control SLESAC contained basically no antibiotic, SL-3.5 contained up to $2 \mu \mathrm{g}$ of thiostrepton per plate and the two mutants SL-Abc and SL-Imp contained 0.07 and $0.05 \mu$ g per plate, respectively. Our results suggest that Imp and Abc are cooperatively involved in increasing the intracellular thiostrepton amount.

\section{Imp and Abc expression is medium-dependent and strain specific}

When SL-3.5 strain was cultured in various liquid or solid media different from $\mathrm{R}_{2} \mathrm{YED}$ (i.e. ONA), the antibiotic activity was never detected (Fig. 6a and data not shown) suggesting that the antibiotic effect could be mediumdependent. Thus, we investigated whether imp and $a b c$ genes were actively transcribed only in $\mathrm{R}_{2} \mathrm{YED}$ medium. Quantitative RT-PCR experiments were performed using total RNA extracted from SL-3.5 mycelium grown for 4 days on $\mathrm{R}_{2}$ YED or ONA agar plates containing thiostrepton. The results clearly demonstrated that both genes are 2,4-fold more transcribed in SL-3.5 when grown in R2YED than in ONA (Fig. 6b). We can surmise that the expression levels of imp and $a b c$ were not enough to determine the uptake of thiostrepton in ONA.

In addition, we investigated whether other streptomycetes showed the same antibacterial effect when they contained these two genes. To this aim, we transformed $S$. coelicolor M145 with ESAC3.5, generating SC-3.5 strain. Its capacity to uptake and maintain thiostrepton was investigated by TLC analysis followed by bioassay of extracts. The findings showed that SC-3.5 extract does not contain thiostrepton (Fig. 6a). In accordance with this result, we found that the transcription level of $a b c$ and imp genes in SC-3.5 was lower than that of SL-3.5, even if $a b c$ transcription was 2-fold more induced in SC-3.5 grown in $\mathrm{R}_{2} \mathrm{YED}$ (Fig. 6b). All together these experiments suggest that thiostrepton uptake is medium-dependent and strain-specific.

\section{Discussion}

Heterologous expression in genetically and physiologically characterized hosts has been used as tool to access the gene products of hard-to-manipulate strains [9]. Streptomyces species offer many potential advantages as 

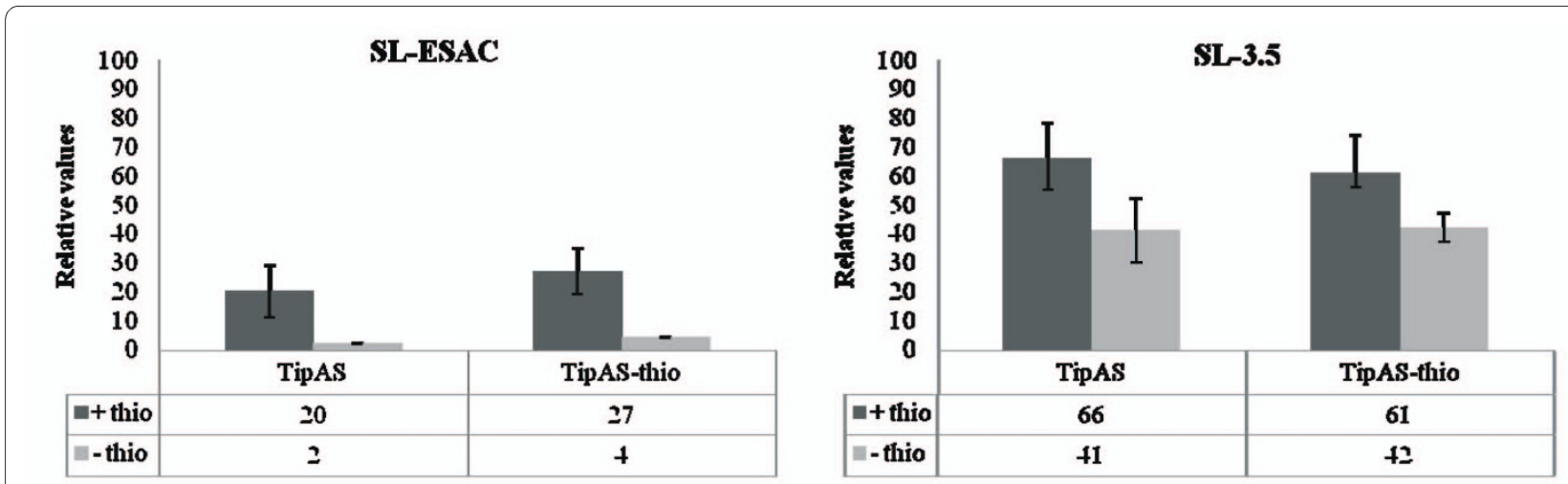

Figure 4 Percentage volume of free and thiostrepton-bound TipAS forms. SL-ESAC and SL-3.5 strains were grown with thiostrepton (+ thio) and for II passages without thiostrepton (- thio).

hosts for the heterologous expression of secondary metabolite genes and we previously have successfully demonstrated heterologous expression of actinomycete genes in S. lividans [12].

At the beginning of this work, we were interested to find new antibacterial activities against Gram-positive bacteria by screening a Planobispora rosea ESAC library in Streptomyces lividans [11]. In particular, we isolated the clone SL-40 containing a $P$. rosea $40 \mathrm{~kb}$ fragment with antibacterial activity against Gram-positive bacteria. Sequencing and BLAST analysis of the insert revealed 30 genes not directly relatable to genes from secondary metabolism biosynthesis. Finally, the sub-cloning revealed that a $3.5 \mathrm{~kb}$ fragment encoding a membrane protein (Imp) and an $\mathrm{ABC}$ transporter (Abc), whose role is unknown in Planobispora, was attributed to the antibacterial effects.

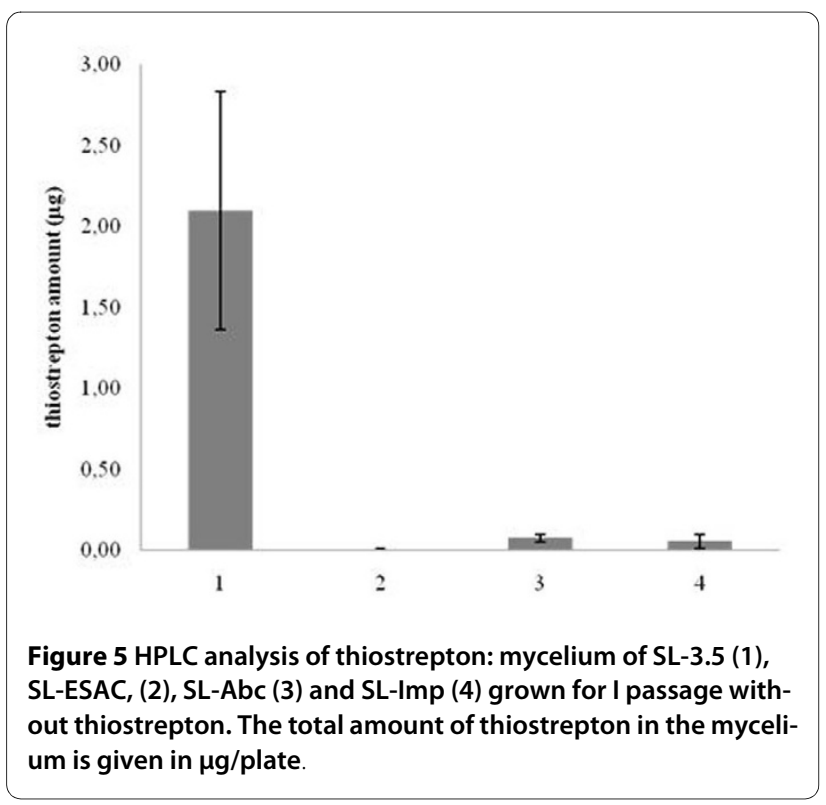

The presence of many cryptic or silent gene clusters in Streptomycetes [16-18] on one hand and the evidence that the two membrane proteins were both implicated in the antibacterial activity on the other hand suggested that the activity was due to an interaction between Planobispora cloned genes and S. lividans genes.

Similar effects have been described by Rondon et al. [19], who found that an antibacterial activity was determined by a membrane protein heterologously expressed in $E$. coli from a metagenomic library and their results

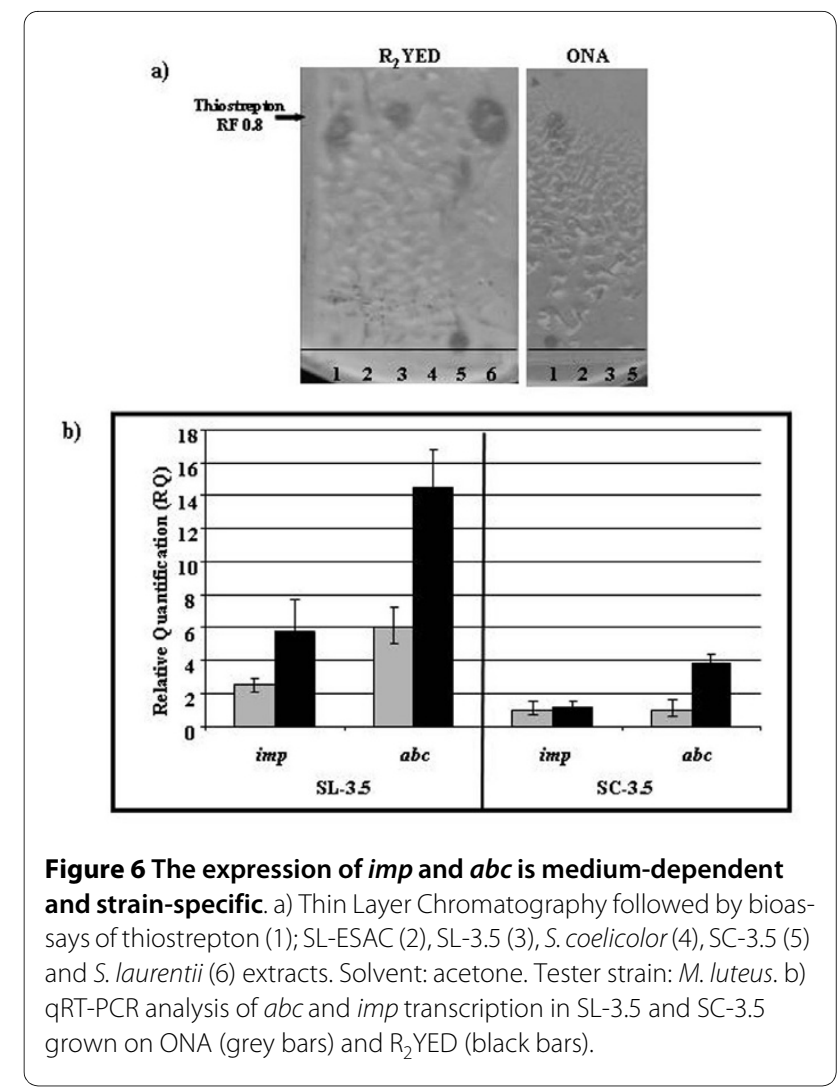


suggested that this protein caused an effect on the host cell.

Unexpectedly, HPLC-ESI-mass spectrometry and HPLC-UV-DAD unambiguously showed that the antibacterial activity was due to thiostrepton, antibiotic used for recombinant clones selection. Furthermore, our findings revealed that a de novo thiostrepton synthesis had not occurred in S. lividans (Fig. 2) but strongly suggested that SL-3.5 antibacterial activity was caused by increased intracellular amounts of thiostrepton (Fig. 4) and its subsequent release in thiostrepton-free medium (Fig. 3). This effect could be explained by an increased uptake of thiostrepton from the medium, particularly triggered by expression of Imp and Abc (in addition to a entry by diffusion). imp and $a b c$ genes were found transcribed in a medium-dependent manner in S. lividans; in fact, their expression level was high enough to support the bioactivity only when the strain was grown in $\mathrm{R}_{2}$ YED medium (Fig. 6b). Further analyses have to be carried out to elucidate the biological mechanism.

In the course of our experiments, the thiostrepton biosynthetic cluster of the producer strain Streptomyces laurentii was sequenced [5] and its absence in the SL-3.5 strain was in agreement with our results (Fig. 2).

Although best known as an inhibitor of protein synthesis, thiostrepton is also a potent activator of gene expression in Streptomyces lividans, i.e. it activates the expression of the so-called thiostrepton-induced proteins (TipAS and TipAL), whose role is still unknown. In the absence of added cofactors, thiostrepton was reported to form a highly stable complex with TipAL and TipAS in aqueous solution, which cannot be dissociated by denaturants such as SDS, urea, or disulfide reducing agents [20]. Our DIGE-analysis showed that large amounts of thiostrepton enter the cell, induce TipA expression and bind this protein, as demonstrated by the abundance of TipAS and TipAS-thio in the mycelium grown also in the absence of thiostrepton (Fig. 5). Thereby, the strain needs to grow for many generations in an antibiotic free medium in order to dilute the thiostrepton intracellular overstock in a appreciably way.

Moreover, the role of thiostrepton is not well understood; it is hypothesized that active thiopeptides are not only antibiotics but may serve as, or perhaps mimic, a signal molecule for physiological development or quorumsensing in bacteria [1]. It was suggested that antibiotics are not only bacterial weapons for fighting competitors but also signaling molecules that may regulate the homeostasis of microbial communities [21]. For example, didehydroamino acids of thiostrepton are highly reactive toward low molecular weight thiols that maintain the cytoplasmic redox potential [22] and it seems likely that thiostrepton levels routinely used for selection of $t s r$-containing vectors could reduce the concentrations of these compounds and thereby disrupt overall cellular thiol-balance.

The expression of the two putative $P$. rosea membrane proteins, responsible for increasing intracellular amounts and storage of thiostrepton in S. lividans, causes a false antibiotic production, that creates errors in the interpretation of clone library screening results.

Our results showed that $S$. coelicolor containing imp and $a b c$ does not uptake thiostrepton, probably since the genes are transcribed at a low level (Fig. 6b). We can speculate that in S. lividans Imp and Abc serve as receptors or channels to up-take molecules such as thiostrepton. Notwithstanding, further analyses are necessary to investigate their role in P. rosea and in other heterologous hosts, as well.

\section{Conclusion}

The heterologous gene expression has to be considered as an useful biotechnological tool not only for new antibiotic discovery but also to study gene function in amenable hosts. However, this work might result in the reconsideration of a careful screening of a genomic library directed to find new antibacterial substances especially when genes encoding membrane proteins are cloned and antibiotics with possible biological effects interfering with the physiological activity of the host, as thiostrepton, are used as clone selection markers.

\section{Methods \\ Reagents}

Thiostrepton was purchased from Fluka (Buchs, Switzerland).

\section{Strains, plasmids and growth conditions}

The bacterial strains and plasmids used in this study are listed in Table 1. Micrococcus luteus and Bacillus subtilis were used for bioassays. Four clones (SL-40, $-85,-48$ and $120)$ were derived from an ESAC genomic library of $P$. rosea [11]. S. lividans strains were cultured in the following media: MG, JM, $\mathrm{R}_{2}$ YED, ONA, TSB, Rare3 and R3 [14,23], according to Puglia et al. [24]. The SL clones were selected using thiostrepton $\left(50 \mu \mathrm{g} \mathrm{ml}^{-1}\right)$.

\section{Subcloning in E. coli and Streptomyces strains}

Plasmid DNA, extracted from EC-40 by alkaline lysis method [25] and digested with $B a m H I$, was ligated to BamHI-digested and dephosphorylated ESAC vector and the ligation mixture was used to transform Escherichia coli ElectroMAX DH10B cells, as described in Alduina et al., 2005 [12]. Streptomyces protoplast formation, transformation and regeneration were carried out according to the methods of Kieser et al. [14]. Southern hybridization was performed according to standard protocols [25]. DNA fragments used as probes were labelled with $[\alpha-$ 
Table 1: Strains and plasmids.

\begin{tabular}{|c|c|c|}
\hline Strain/Plasmid & Genotype/Description & Source/Reference \\
\hline \multicolumn{3}{|l|}{ Bacterial strains } \\
\hline Planobispora rosea ATCC53773 & & Biosearch Italia SpA, Gerenzano \\
\hline Streptomyces lividans ZX7 & pro-2 str-6 rec-46 $\triangle$ dnd HAU3SLP2- SLP3- & John Innes Centre, Norwich, UK \\
\hline Streptomyces laurentii & & $\begin{array}{l}\text { German Collection of Microorganisms and Cell } \\
\text { Cultures, DSMZ GmbH, Braunschweig, Germany }\end{array}$ \\
\hline Streptomyces coelicolor M145 & & John Innes Centre, Norwich, UK \\
\hline Escherichia coli $\mathrm{DH} 10 \mathrm{~B}$ & $\begin{array}{l}\text { F- mcrA } \triangle \text { (mrr- hsdRMS-mcr BC) } \triangle 80 d l a c Z D M 15 \\
\Delta l a c X 74 \text { deoR recA1 endA1 araD139 }(\text { (ara, leu) } \\
7697 \text { galU GalK D' rpsL nupG }\end{array}$ & Life Technologies, Gibco BRL. \\
\hline $\mathrm{EC}-40$ & E. coli containing ESAC40 & Alduina et al., 2003 \\
\hline SC-3.5 & S. coelicolor containing ESAC3.5 & This study \\
\hline SL-ESAC & S. lividans containing ESAC & Alduina et al., 2003 \\
\hline SL-40 & S. lividans containing ESAC40 & Alduina et al., 2003 \\
\hline SL-3.5 & S. lividans containing ESAC3.5 & This study \\
\hline SL-Abc & S. lividans containing ESACAbc & This study \\
\hline SL-Imp & S. lividans containing ESACImp & This study \\
\hline $\mathrm{SL}-\mathrm{Abc} / \mathrm{Imp}$ & S. lividans containing ESACAbc and pNImp & This study \\
\hline SL-Imp/Abc & S. lividans containing ESACImp and pNAbc & This study \\
\hline \multicolumn{3}{|l|}{ Plasmids } \\
\hline $\mathrm{pUC}$ & pUC18 & \\
\hline pBS & pBluescript & \\
\hline ESAC & Streptomyces-E. coli Artificial Chromosome & Sosio et al., 2002 \\
\hline ESAC40 & ESAC containing a P.rosea $40 \mathrm{~kb}$ fragment & Alduina et al., 2003 \\
\hline ESAC3.5 & $\begin{array}{l}\text { ESAC containing a P.rosea } 3.5 \mathrm{~kb} \text { fragment with } \\
a b c \text { and } i m p \text { genes }\end{array}$ & This study (fig.1) \\
\hline ESACAbc & ESAC3.5/imp:::pBS & This study (fig.1) \\
\hline ESACImp & ESAC3.5/abc::pUC & This study (fig.1) \\
\hline pN702GEM3 & & Fernández-Abalos et al., 2003 \\
\hline pNAbc & pN702 containing $a b c$ & This study \\
\hline pNImp & pN702 containing imp & This study \\
\hline
\end{tabular}

32P]-CTP by the Rediprime II $^{\mathrm{m}}$ Random Prime Labelling System (Amersham Pharmacia Biotech, UK).

\section{Sequencing of the $\mathbf{4 0} \mathrm{kb}$ DNA fragment from ESAC40}

DNA sequencing was performed by MWG Biotech Laboratories (Ebersberg, Germany). Open reading frame (ORF) analysis was performed using Swiss prot protein knowledge base. Putative orfs were analyzed using BLAST against the NCBI non redundant protein database. The GenBank sequence accession number of the Planobispora rosea $40 \mathrm{~kb}$ DNA fragment is EU908202.

\section{Disruption of imp and abc}

The imp and $a b c$ disruption mutants were obtained by inserting pBluescript and pUC18 into the unique sites $A p a \mathrm{I}$ and $S a c \mathrm{I}$ site of ESAC3.5, respectively. The resulting ESAC $a b c$ and ESACimp plasmids were used to transform S. lividans protoplasts, generating SL-Abc and SL-Imp clones, respectively. Genomic DNA extracted from these clones was digested with BamHI and analyzed by Southern hybridization using the $3.5 \mathrm{~Kb} B a m \mathrm{HI}$ fragment from ESAC3.5, containing both orfs, as a probe. As expected, two bands (6.5 and $6.2 \mathrm{~Kb}$, respectively) were obtained for 
SL-Abc and SL-Imp (Fig. 1B, lane 5 and 6), while a $3.5 \mathrm{~kb}$ band was obtained for SL-3.5 (Fig. 1B, lane 1).

\section{Complementation of imp and abc mutations}

A HindIII fragment containing imp and a BglII fragment containing $a b c$, derived from ESAC3.5, were ligated into HindIII and BamHI sites of pN702GEM3 [26] to obtain pNImp and pNAbc plasmids, respectively. These plasmids were introduced by protoplast transformation in SL-Abc and SL-Imp to generate complementation strains, isolated under apramycin selection $\left(100 \mu \mathrm{g} / \mathrm{ml}^{-1}\right)$.

\section{Antibacterial assays and pre-purification of compounds}

Antibacterial assays were carried out as follows: $5 \mathrm{ml}$ of LB soft agar containing $100 \mu \mathrm{l}$ of $M$. luteus suspension in water $\left(\mathrm{OD}_{600}\right.$ of 1-1.2) were overlaid on $\mathrm{LB}$ agar plates. $\mathrm{R}_{2}$ YED plugs of S. lividans strains, grown for four days at $30^{\circ} \mathrm{C}$, were put on the Micrococcus overlay. Plates were then incubated overnight at $37^{\circ} \mathrm{C}$ and scored for activity by looking for an inhibition zone in the M. luteus lawn. The protocols reported in Hwang et al., 2003 [27] and Adamis et al., 1990 [28] were used for the detection of actinorhodin, undecylprodigiosin and calcium-dependent antibiotic. To identify the antibiotic substance, the methanolic extract of 20 agar plates for each analysed strain was dried in vacuo and dissolved in $1 \mathrm{l}$ of distilled water. After XAD-chromatography (XAD-16 material, $300 \mathrm{ml}$ column; 2 wash steps: $11 \mathrm{H}_{2} \mathrm{O}, 1110 \% \mathrm{MeOH}$; elution with $500 \mathrm{ml}$ acetone) and evaporation of the solvent, the extract was dissolved in EtOAc/Acetone/ $\mathrm{H}_{2} \mathrm{O}(1 / 1 / 1)$ and both phases were analysed by HPLC-ESI-MS.

\section{HPLC-ESI-mass spectrometry}

HPLC-ESI-MS and HPLC-UV-DAD experiments were carried out with a Q-Trap 2000 mass spectrometer from Applied Biosystems Deutschland GmbH (Darmstadt, Germany) (HPLC-ESI-MS), equipped with an 1100 series capillary HPLC system (HPLC-ESI-MS) or a 1100 series HPLC system (HPLC-UV-DAD) from Agilent Technologies Deutschland AG (Waldbronn, Germany) fitted with a Luna $3 \mathrm{u}$ C18(2) $100 \AA$, $50 \times 1 \mathrm{~mm}$ column (HPLC-ESIMS) or $50 \times 4.5 \mathrm{~mm}$ column (HPLC-UV-DAD) purchased from Phenomenex, Aschaffenburg, Germany. Gradient 1: $25 \%$ B to $100 \%$ B in 10 min, gradient 2: 5\% B to $100 \% \mathrm{~B}$ in $10 \mathrm{~min}$ (A: water, HPLC-MS grade, $0.1 \%$ formic acid, analytical grade; B: acetonitrile, HPLC-MSgrade, $0.1 \%$ formic acid, analytical grade, all solvents were purchased from Carl Roth GmbH \& Co, Karlsruhe, Germany).

The upper phase of SL-3.5 water:acetone:ethyl acetate (1:1:1) extract contained a large peak in HPLC-ESI-MS and HPLC-UV-DAD that was absent in SL-ESAC. Furthermore, experiments confirmed its identity to thiostrepton purchased from Fluka (data not shown).
Thiostrepton elutes at $7.0 \mathrm{~min}$ (HPLC-ESI-MS, gradient 1), $8.9 \mathrm{~min}$ (HPLC-ESI-MS, gradient 2) and $7.1 \mathrm{~min}$ (HPLC-UV-DAD, gradient 2), respectively and has a molecular mass of $[\mathrm{M}+\mathrm{H}]^{+}=1664.5$.

\section{Thin Layer Chromatography (TLC) and Feeding experiments}

$1 \mathrm{ml}$ of pre-culture in JM medium of SL-ESAC, SL-3.5, SC-3.5, Streptomyces coelicolor and S. laurentii were streaked on $\mathrm{R}_{2}$ YED medium and incubated at $30^{\circ} \mathrm{C}$ for four days before freezing at $-20^{\circ} \mathrm{C}$. The frozen mixture was added to $30 \mathrm{ml}$ of methanol. An aliquot of 10-fold concentrated methanol extract was extracted with a mixture of water:acetone:ethyl acetate (1:1:1) and the upper phase was analyzed by Thin Layer Chromatography (TLC). TLC plates were developed in acetone and used for the bioassay. $0.050 \mu \mathrm{g} / \mu \mathrm{l}$ of thiostrepton in water:acetone:ethylacetate 1:1:1 were used as control.

In feeding experiments the isotopically labelled substrate $(\mathrm{R}, \mathrm{S})-\left[3^{-14} \mathrm{C}\right]$ cysteine $(30 \mu \mathrm{Ci} \mathrm{ml}-1)$ was added to $\mathrm{R}_{2}$ YED medium and its presence in the extracts was detected by autoradiographic exposition of the TLC plate after acetone development.

\section{Analysis of thiostrepton in the mycelium of SL-3.5}

SL-3.5, S. laurentii (a thiostrepton producer, positive control) and SL-ESAC (negative control) were each grown on $\mathrm{R}_{2}$ YED agar plates containing thiostrepton $(50 \mu \mathrm{g} / \mathrm{ml})$. After 4 days mycelium from all the three strains was collected and transferred to thiostrepton-free $\mathrm{R}_{2}$ YED agar plates (passage I). Mycelium from this passage was subsequently transferred to new thiostrepton-free R2YED agar plates (passage II). This procedure was repeated 10 times. To facilitate mycelium isolation, cellophane was placed between the agar and the growing Streptomyces strains. After passages I, V and X of each strain, 0.16-0.17 $\mathrm{g}$ of mycelium were separated from agar and resuspended in 8 $\mathrm{ml}$ of distilled water. The suspension was sonicated three times on ice (15 sec in Vibra cell-output control 4) and the lysates were centrifuged for $10 \mathrm{~min}$ at 3000 r.p.m. Thiostrepton present in the pellet was extracted using 2 $\mathrm{ml}$ of DMSO and $5 \mu \mathrm{l}$ of this extract were analysed by bioassay on $M$. luteus and visualization using an UV lamp (302 $\mathrm{nm}$ ). The remaining solution was analyzed by HPLC-ESI-MS: the samples were freeze-dried and dissolved in $200 \mu \mathrm{l}$ DMSO, centrifuged and $4 \mu \mathrm{l}$ of the clear supernatant were injected into the HPLC-ESI mass spectrometer. The mass range during data acquisition was set to $m / z 1600-1680$ and the peak at $t_{\mathrm{R}}=8.9 \mathrm{~min}$ and $[\mathrm{M}+\mathrm{H}]^{+}=1664,5 \mathrm{Da}$ corresponding to thiostrepton was integrated using the quantification tool in the MS-software Analyst 1.4.2 from Applied Biosystems. Prior to thiostrepton quantification a calibration curve was established using external samples of thiostrepton dissolved in 
DMSO in 10 different concentrations between $0.05 \mu \mathrm{g} / \mathrm{ml}$ and $20 \mu \mathrm{g} / \mathrm{ml}\left(\mathrm{y}=2332498.99 \times, \mathrm{R}^{2}=0.97\right)$.

\section{Protein extraction, separation and analysis}

Cells, grown as above, were recovered from cellophane and protein extraction was performed as described previously [11]. Protein concentration was quantified using the Qubit $^{\text {TM }}$ fluorometer (Invitrogen). After dialysis against distilled water at $4^{\circ} \mathrm{C}$ and acetone precipitation at $-20^{\circ} \mathrm{C}$, $300 \mathrm{mg}$ of extracted protein was resuspended in $50 \mathrm{ml}$ of lysis buffer (30 mM TRIS, $7 \mathrm{M}$ urea, $2 \mathrm{M}$ thiourea, 4\% CHAPS) to carry out Two-Dimensional Differential Gel Electrophoresis (2D-DIGE, Amersham).

The $\mathrm{pH}$ of protein extracts was adjusted to $8.5-8.8$ and $75 \mathrm{mg}$ of proteins were labelled with CyDye DIGE fluors (Cy3 for SL-3.5 grown with or without thiostrepton and SL-Abc with thiostrepton; Cy5 for SL-ESAC with or without thiostrepton and SL-Abc without thiostrepton), according to the manufacturer's instruction. A pooled set of internal standards, comprising $25 \mathrm{mg}$ aliquot from each sample, was minimally labelled with Cy2 DIGE fluors. After the labelling, rehydratation buffer was added to obtain $350 \mu$ l (final volume) before IEF separation. Proteins were first separated on $18 \mathrm{~cm}$ IPG strips (non-linear gradient $\mathrm{pH} 3-10$ ) in the ETTAN IPGphor isoelectric focusing system. The IPG strips were rehydrated in IEF buffer containing $0.5 \%(\mathrm{w} / \mathrm{v})$ ampholytes and $1 \%(\mathrm{w} / \mathrm{v})$ bromophenol blue. For protein separation, a $30-\mathrm{V}$ prestep was performed for $10 \mathrm{~h}$, followed by IEF carried out for 74,850 V-h with a maximum voltage of $8,000 \mathrm{~V}$. All the steps were performed at $20^{\circ} \mathrm{C}$ using $50 \mu \mathrm{A}$ per strip. After IEF, the IPG strips were saturated with an equilibration buffer (6 M urea, 30\% (v/v) glycerol, 2\% (w/v) SDS, 0.05 $\mathrm{M}$ TRIS-HCl pH 6.8 and 2\% (w/v) DTE) for $12 \mathrm{~min}$, in order to resolubilize proteins. The thiol groups of Cys were then blocked by substituting DTE with $2.5 \%(\mathrm{w} / \mathrm{v})$ iodoacetamide in the equilibrating buffer. The focused proteins were then separated on $12 \%$ sodium dodecyl polyacrylamide gels (SDS-PAGE) at $16^{\circ} \mathrm{C}$ in a Hoefer Dalt vertical system, using a maximum setting of $40 \mu \mathrm{A}$ and $110 \mathrm{~V}$ per gel. Two-dimensional gel analysis was performed using the DIGE algorithms of ImageMaster 2D platinum software (v. 6.01, GE Healthcare Biosciences) according to the manufacturer's instructions. Spot detection was performed automatically using the auto-detection device. Spot quantification was calculated as the volume of the spots (i.e. integration of optical density over the spot area). Spot volumes were normalized (\%Vol) to the sum of the volume of all spots detected on each gel by the software. To better visualize the results, the relative values were reported as $\% \mathrm{Vol} \times 100$.

Total RNA isolation, RT-PCR analysis, and real-time RT-PCR Collected mycelium of SL-3.5 and SC-3.5 grown on $\mathrm{R}_{2}$ YED and ONA agar plates was broken by using $1 \mathrm{mg}$ of lysozyme/ml in P-buffer and total RNA was extracted by using the RNeasy midi-kit (QIAGEN) and following the procedures reported in Alduina et al., 2007 [29]. As control of RNA quality, a reverse transcription-PCR (RTPCR) with $0.1 \mu \mathrm{g}$ of total RNA and primer pairs internal to $\operatorname{hrdB}$ (5'-cgtcgagggtcttcggctg-3' and 5'-cgcgagccatctcgctgc-3') was carried out using a Superscript OneStep RT-PCR kit (Invitrogen) and the conditions indicated by the supplier. A negative control with Taq polymerase and without reverse transcriptase was included to assess the absence of chromosomal DNA.

Expression of imp and $a b c$ was analyzed quantitatively by real-time RT-PCR using the Applied Biosystems 7300 real-time PCR system (Applied Biosystems). Specific primers internal to imp (5'-tgccagcgcgtacaggtgtcg-3' and $5^{\prime}$-cgcgggtcttggtctggctgt- $\left.3^{\prime}\right)$ and $a b c$ (5'-cgtgcctggccettcatcgac-3' and 5'-ggcctggcagatcatcgtgg-3') genes were designed using the web-based tool GeneTool ${ }^{\mathrm{TM}}$ [30]. For cDNA synthesis, $4 \mu \mathrm{g}$ for each RNA were used as template in a $50 \mu \mathrm{l}$ reaction volume employing High Capacity cDNA Archive Kit (Applied Biosystems, USA) according to the manufacturer's instructions. PCR cycling was performed at $25^{\circ} \mathrm{C}$ for 10 minutes, $37^{\circ} \mathrm{C}$ for 120 minutes and then 5 minutes at $85^{\circ} \mathrm{C}$.

$2.5 \mu \mathrm{l}$ of each sample were used in the quantitative PCR reaction with $\mathrm{SYBR}^{\circ}$ Green PCR Master Mix (Applied Biosystems, USA) according to the manufacturer's instructions. Each $25 \mu \mathrm{l}$ reaction contained $10 \mathrm{pmol}$ of forward and reverse primers.

The PCR was performed under the following conditions: $2 \mathrm{~min}$ at $50^{\circ} \mathrm{C}$ and $10 \mathrm{~min}$ at $95^{\circ} \mathrm{C}$, followed by 40 cycles of $15 \mathrm{~s}$ at $95^{\circ} \mathrm{C}$ and $1 \mathrm{~min}$ at $60^{\circ} \mathrm{C}$. Eventually, a dissociation reaction was performed with the following conditions: a 1-min step with a temperature gradient increase of $1^{\circ} \mathrm{C}$ per step from 55 to $99^{\circ} \mathrm{C}$. This last reaction allowed the melting curve of the PCR products and, consequently, to determine their specificity. A negative control (distilled water) was included in all real-time PCR assays, and each experiment was performed in triplicate. The hrdB (5'-cgtcgagggtcttcggctg-3' and 5'-cgcgagcccatctcgctgc-3') gene was used as an internal control to quantify the relative expression of target genes.

\section{Competing interests}

The authors declare that they have no competing interests.

\section{Authors' contributions}

AG carried out the construction of the mutants, feeding and real-time RT-PCR experiments and DIGE analysis and wrote the draft manuscript. RA carried out Southern analysis, participated in the design of the experiments and helped to draft the manuscript. EG carried out the chemical analysis and helped to draft the manuscript. VDC carried out the construction of S. lividans subclones. RDS participated in the design of experiments and revised the manuscript. AMP conceived the study and participated in its design and coordination and revised the manuscript.

All authors read and approved the final manuscript. 


\section{Acknowledgements}

This work was supported by a grant of the European Union (LSHMCT-2004005224) given to AMP and RDS. We thank Valentina Bravatà for technical assistance and Giuseppe Gallo for helpful advice on methods for DIGE analysis.

\section{Author Details}

'Dipartimento di Biologia Cellulare e dello Sviluppo, Università di Palermo, Viale delle Scienze, Ed.16, 90128 Palermo, Italy, ${ }^{2}$ Technische Universität Berlin, Strasse des 17. Juni 124, 10623 Berlin, Germany and ${ }^{3}$ Fondazione RiMed, Piazza Sett'Angeli 10, 90134 Palermo, Italy

Received: 23 February 2010 Accepted: 9 June 2010

Published: 9 June 2010

\section{References}

1. Holmes DJ, Caso JL, Thompson CJ: Autogenous transcriptional activation of a thiostrepton-induced gene in Streptomyces lividans. EMBO J 1993, 12:3183-3191.

2. Chiu ML, Folcher M, Katoh T, Puglia AM, Vohradsky J, Yun BS, Seto H, Thompson CJ: Broad spectrum thiopeptide recognition specificity of the Streptomyces lividans TipAL Protein and its role in regulating gene expression. JBC 1999, 274(29):20578-20586.

3. Murakami T, Holt TG, Thompson CJ: Thiostrepton-induced gene expression in Streptomyces lividans. J Bacteriol 1989, 171:1459-1466.

4. Yun BS, Hidaka T, Kuzuyama T, Seto H: Thiopeptide non-producing Streptomyces species carry the tipA gene: a clue to its function. $J$ Antibiot 2001, 54(4):375-378.

5. Kelly WL, Pan L, Li C: Thiostrepton biosynthesis: Prototype for a new family of bacteriocins. J Am Chem Soc 2009, 131(12):4327-4334.

6. Liao R, Duan L, Lei C, Pan H, Ding Y, Zhang Q, Chen D, Shen B, Yu Y, Liu W: Thiopeptide biosynthesis featuring ribosomally synthesized precursor peptides and conserved posttranslational modifications. Chem Biol 2009, 16(2):141-147.

7. Morris RP, Leeds JA, Naegeli HU, Oberer L, Memmert K, Weber E, LaMarche MJ, Parker CN, Burrer N, Esterow S, Hein AE, Schmitt EK, Krastel P: Ribosomally synthesized thiopeptide antibiotics targeting elongation factor Tu. J Am Chem Soc 2009, 131(16):5946-5955.

8. Arndt HD, Schoof S, Lu JY: Thiopeptide antibiotic biosynthesis. Angew Chem Int Ed Engl 2009, 48(37):6770-6773.

9. Zhang $\mathrm{H}$, Wang Y, Pfeifer BA: Bacterial hosts for natural product production. Mol Pharm 2008, 5(3):472.

10. Alduina R, Ferraro C, Giardina A, Di Caro V, Sosio M, Donadio S, Puglia AM: Bacterial artificial chromosome libraries of antibiotic-producing actinomycetes. In Applications of Genomics and Proteomics for Analysis of Bacterial Biological Warfare Agents Volume 352. Edited by: Del Vecchio V, Krcmery V. NATO Science Series.; 2003:111-121.

11. Alduina R, De Grazia S, Dolce L, Salerno P, Sosio M, Donadio S, Puglia AM: Artificial chromosome libraries of Streptomyces coelicolor A3(2) and Planobispora rosea. FEMS Microbiol Lett 2003, 218:181-186.

12. Alduina R, Giardina A, Gallo G, Renzone G, Ferraro C, Contino A, Scaloni A, Donadio S, Puglia AM: Expression in Streptomyces lividans of Nonomuraea genes cloned in an artificial chromosome. App/ Microbiol Biotechnol 2005, 68(5):656-662.

13. Selva E, Beretta G, Montanini M, Saddler GS, Gastaldo L, Ferrari P, Lorenzetti R, Ripamonti F, Goldstein BP, Berti M, Montanaro L, Denaro M: Antibiotic GE2270 a: a novel inhibitor of bacterial protein synthesis. Isolation and characterization. J Antibiot 1991, 44(7):693-701.

14. Kieser T, Bibb MJ, Buttner MJ, Chater KF, Hopwood DA: Pratical Streptomyces genetics. John Innes Centre, Norwich 2000.

15. Chiu ML, Viollier PH, Katoh T, Ramsden JJ, Thompson CJ: Ligand-induced changes in the Streptomyces lividans TipAL protein imply an alternative mechanism of transcriptional activation for MerR-like proteins. Biochemistry 2001, 40(43):12950-12958.

16. Bentley SD, Chater KF, Cerdeño-Tárraga AM, Challis GL, Thomson NR, James KD, Harris DE, Quail MA, Kieser H, Harper D, Bateman A, Brown S, Chandra G, Chen CW, Collins M, Cronin A, Fraser A, Goble A, Hidalgo J, Hornsby T, Howarth S, Huang CH, Kieser T, Larke L, Murphy L, Oliver K, O'Neil S, Rabbinowitsch E, Rajandream MA, Rutherford K, Rutter S, Seeger K, Saunders D, Sharp S, Squares R, Squares S, Taylor K, Warren T, Wietzorrek A, Woodward J, Barrell BG, Parkhill J, Hopwood DA: Complete genome sequence of the model actinomycete Streptomyces coelicolor A3(2). Nature 2002, 417(6885):141-147.

17. Ikeda H, Ishikawa J, Hanamoto A, Shinose M, Kikuchi H, Shiba T, Sakaki Y, Hattori M, Omura S: Complete genome sequence and comparative analysis of the industrial microorganism Streptomyces avermitilis. Nat Biotechnol 2003, 21(5):526-531.

18. Ohnishi Y, Ishikawa J, Hara H, Suzuki H, Ikenoya M, Ikeda H, Yamashita A, Hattori M, Horinouchi S: Genome sequence of the streptomycinproducing microorganism Streptomyces griseus IFO 13350. J Bacteriol 2008, 190(11):4050-4060.

19. Rondon MR, August PR, Bettermann AD, Brady SF, Grossmann TH, Liles MR, Loiacono KA, Lynch BA, MacNeil IA, other authors: Cloning the soil metagenome: a strategy for accessing the genetic and functional diversity of uncultured microorganism. App Environ Microbiol 2000, 66:2541-2547.

20. Chiu ML, Folcher M, Griffin P, Holt T, Thompson CJ: Characterization of the covalent binding of Thiostrepton-induced protein from Streptomyces lividans. Biochemistry 1996, 35:2332-2341.

21. Linares JF, Gustafsson I, Baquero F, Martinez JL: Antibiotics as intermicrobial signaling agents instead of weapons. PNAS 2006, 113(51):19484-19489.

22. Newton GL, Bewley CA, Dwyer TJ, Horn R, Aharonowitz Y, Cohen G, Davies J, Faulkner DJ, Fahey RC: The structure of U17 isolated from Streptomyces clavuligerus and its properties as antioxidant thiol. Eur J Biochem 1995, 230:821-825.

23. Sosio M, Giusino F, Cappellano C, Bossi E, Puglia AM, Donadio S: Artificial chromosomes for antibiotic-producing actinomyces. Nat Biotechnol 2000, 18:343-345

24. Puglia AM, Vohradsky J, Thompson CJ: Developmental control of the heat-shock stress regulon in Streptomyces coelicolor. Mol Microbiol 1995, 17(4):737-746.

25. Sambrook J, Fritsh EF, Maniatis T: Molecular cloning: a laboratory manual. 2nd edition. Cold Spring Harbor, NY: Cold Spring Harbor Laboratory; 1989

26. Fernández-Abalos JM, Reviejo V, Díaz M, Rodriguez S, Leal F, Santamaría RI: Postranslational processing of the xylanase Xys $1 \mathrm{~L}$ form Streptomyces halstedii JM8 is carried out by serine proteases. Microbiology-SGM 2003, 149:1623-1632.

27. Hwang YS, Kim ES, Biró S, Choi CY: Cloning and analysis of a DNA fragment stimulating avermectin production in various Streptomyces avermitilis species. App Environ Microbiol 2003, 69:1263-1269.

28. Adamis T, Riggle $\mathrm{P}$, Champness W: Mutation in a new Streptomyces coelicolor locus with globally block antibiotic biosynthesis but not sporulation. J Bacteriol 1990, 172:2962-2969.

29. Alduina R, Lo L Piccolo, D'Alia D, Ferraro C, Gunnarsson N, Donadio S, Puglia AM: Phosphate-controlled regulator for the biosynthesis of the dalbavancin precursor A40926. J Bacterio/ 2007, 189:8120-9.

30. Wishart DS, Stothard P, Van Domselaar GH: PepTool and GeneTool: platform-independent tools for biological sequence analysis. Methods Mol Biol 2000, 132:93-113.

\section{doi: 10.1186/1475-2859-9-44}

Cite this article as: Giardina et al., Two heterologously expressed Planobispora rosea proteins cooperatively induce Streptomyces lividans thiostrepton uptake and storage from the extracellular medium Microbial Cell Factories 2010, 9:44 